\title{
YopP-Expressing Variant of $Y$. pestis Activates a Potent Innate Immune Response Affording Cross-Protection against Yersiniosis and Tularemia
}

\author{
Ayelet Zauberman, Yehuda Flashner, Yinon Levy, Yaron Vagima, Avital Tidhar, Ofer Cohen, Erez Bar- \\ Haim, David Gur, Moshe Aftalion, Gideon Halperin, Avigdor Shafferman, Emanuelle Mamroud*
}

Department of Biochemistry and Molecular Genetics, Israel Institute for Biological Research, Ness-Ziona, Israel

\begin{abstract}
Plague, initiated by Yersinia pestis infection, is a rapidly progressing disease with a high mortality rate if not quickly treated. The existence of antibiotic-resistant $Y$. pestis strains emphasizes the need for the development of novel countermeasures against plague. We previously reported the generation of a recombinant $Y$. pestis strain (Kim53 $\Delta \mathrm{J}+\mathrm{P}$ ) that over-expresses $Y$. enterocolitica YopP. When this strain was administered subcutaneously to mice, it elicited a fast and effective protective immune response in models of bubonic, pneumonic and septicemic plague. In the present study, we further characterized the immune response induced by the Kim53 $3 \mathrm{~J}+\mathrm{P}$ recombinant strain. Using a panel of mouse strains defective in specific immune functions, we observed the induction of a prompt protective innate immune response that was interferon- $\gamma$ dependent. Moreover, inoculation of mice with $Y$. pestis Kim53 $\Delta \mathrm{J}+\mathrm{P}$ elicited a rapid protective response against secondary infection by other bacterial pathogens, including the enteropathogen $Y$. enterocolitica and the respiratory pathogen Francisella tularensis. Thus, the development of new therapies to enhance the innate immune response may provide an initial critical delay in disease progression following the exposure to highly virulent bacterial pathogens, extending the time window for successful treatment.
\end{abstract}

Citation: Zauberman A, Flashner Y, Levy Y, Vagima Y, Tidhar A, et al. (2013) YopP-Expressing Variant of Y. pestis Activates a Potent Innate Immune Response Affording Cross-Protection against Yersiniosis and Tularemia. PLoS ONE 8(12): e83560. doi:10.1371/journal.pone.0083560

Editor: Yousef Abu Kwaik, University of Louisville, United States of America

Received October 22, 2013; Accepted November 13, 2013; Published December 16, 2013

Copyright: (c) 2013 Zauberman et al. This is an open-access article distributed under the terms of the Creative Commons Attribution License, which permits unrestricted use, distribution, and reproduction in any medium, provided the original author and source are credited.

Funding: This work was supported by the Israel Institute for Biological Research funds, grand number SB/5112-50. The funders had no role in study design, data collection and analysis, decision to publish, or preparation of the manuscript.

Competing Interests: The authors have declared that no competing interests exist.

*E-mail: emmym@iibr.gov.il

\section{Introduction}

Plague is a fatal disease that has caused millions of deaths in three world pandemics, and it remains a public health concern in some regions of the world [1] [2]. The disease, initiated by infection with the bacterial pathogen Yersinia pestis, is manifested in two main forms: pneumonic plague and bubonic plague. Primary pneumonic plague is the more fatal form of the disease, although it is less frequent in nature; it results from the inhalation of $Y$. pestiscontaining droplets or aerosols. Bubonic plague develops following transmission of the pathogen from rodent reservoirs to humans by infected fleas [1]. Bacteria migrate through the cutaneous lymphatics to regional lymph nodes, where they multiply rapidly, followed by colonization of the spleen and liver and, finally, bacteremia and systemic dissemination to other tissues. Nonspecific symptoms start several days after infection and are followed by the development of swollen lymph nodes, which are termed buboes [3].

The medical treatment of plague should be initiated as early as possible to be effective. Antibiotic administration is the wellestablished first-line therapy against plague. However, the existence of antibiotic-resistant $\Upsilon$. pestis strains highlights the need for the development of new therapeutic tools [4]. Three main strategies have been adopted to achieve this goal: a. Search for anti-infective agents that can inhibit the activity of specific essential virulence systems or virulence factors. These agents include small molecules that target the type III secretion system (T3SS) apparatus and specific T3SS effectors [5-8]. b. Development of subunit vaccine candidates that extend the spectrum of the immune response generated by the F1/LcrV-based vaccines are also promising $[9,10]$. c. Attenuated $Y$. pestis strains have been used in an effort to reveal potential targets for new therapeutic measures or to elucidate elements of the host immune system that are required for the generation of protective immunity against plague [11-16]

We have recently shown that a newly engineered strain of $Y$. pestis, Kim $53 \Delta \mathrm{J}+\mathrm{P}$, is dramatically attenuated in a mouse model of bubonic plague $\left(\mathrm{LD}_{50}>10^{7} \mathrm{cfu}\right)$, whereas its virulence is retained in mouse models of pneumonic and systemic plague [17]. This strain was generated by the over-expression of the $Y$. enterocolitica 0:8 T3SS effector YopP in the virulent yopJ-deleted Kimberley53 $r$. pestis strain. YopP is homologous to the endogenous $r$. pestis YopJ; both are known to inhibit the pro-inflammatory response in target host cells [18-20]. However, the recombinant YopPexpressing strain was found to induce cell death more effectively than the wild-type strain, most likely due to differences in YopJ/ YopP translocation efficiency and allelic variations among the genes encoding these effectors $[17,21]$. These results are similar to the findings of Brodsky et al., who demonstrated that the ectopic expression of YopP from $Y$. enterocolitica in $Y$. pseudotuberculosis led to 
attenuation following the oral infection of mice [22]. Interestingly, the $\Upsilon$. pestis $\mathrm{Kim} 53 \Delta \mathrm{J}+\mathrm{P}$ strain induced a rapid and effective systemic immune response that provided protection against subcutaneous (s.c.), intranasal (i.n.) and intravenous challenges with a fully virulent $\Upsilon$. pestis strain [17].

In the present study, we further characterized the rapid immune response induced by the $\mathrm{Kim} 53 \Delta \mathrm{J}+\mathrm{P}$ recombinant $Y$. pestis strain in an effort to uncover a potential new avenue for the development of protective measures against plague. Using immunodeficient mouse strains, we observed the induction of a prompt but transient protective innate immune response that was interferon- $\gamma$ dependent. This response appeared to postpone disease progression and allow the development of adaptive immunity. Consistent with the relatively low specificity characterizing the rapid induction of the innate response, Kim53 $\Delta \mathrm{J}+\mathrm{P}$-infected wild-type mice were also protected from a lethal challenge with the enteropathogen $\Upsilon$. enterocolitica and furthermore with a non-related respiratory pathogen, Francisella tularensis subspecies holarctica LVS.

\section{Materials and Methods}

\section{Bacterial strains}

The bacterial strains used in this study are listed in Table 1. Yersinia strains were routinely grown on brain heart infusion agar (BHIA, Difco) for 48 hours at $28^{\circ} \mathrm{C}$. Ampicillin-resistant $Y$. pestis strains (Kim53pGFP, Kim53 $\Delta \mathrm{J}+\mathrm{P}$ ) were grown on BHIA supplemented with $100 \mu \mathrm{g} / \mathrm{ml}$ ampicillin (Sigma, Israel). Live $F$. tularensis vaccine strain (ATCG 29684) stocks were plated on GCHI agar (GC Medium base [Difco Laboratories] supplemented with 1\% hemoglobin and 1\% Iso-VitaleX [BD, France]).

\section{Ethics statement}

This study was carried out in strict accordance with the recommendation in the Guide for the Care and Use of Laboratory Animals of the National Institute of Health. All animal experiments were performed in accordance with Israeli law and were approved by the Ethics Committee for animal experiments at the Israel Institute for Biological Research. (Permit Number: IACUCIIBR M-32-2-09, IACUG-IIBR M-57-09, IACUG-IIBR M-58-11 and IACUC-IIBR M-62-11, IAGUC-IIBR M-23-12, IACUCIIBR M-26-13). During the experiments mice were monitored daily. Humane endpoints were used in our survival studies. Mice exhibiting loss of the righting reflex were euthanized by cervical dislocation. Analgesics were not used as they may have affected the experimental outcomes of the studies.

\section{Animals}

Five- to six-week-old female OF1 mice were purchased from Charles River Laboratories (IFFA CREDO S.A., France). Female C67BL/6J, $\mu \mathrm{MT}$ (B6.129S2-Ighm $\left.{ }^{\mathrm{tm} 1 \mathrm{Cgn}} / \mathrm{J}\right), \mathrm{RAG}^{-/-}$(B6;129S7-

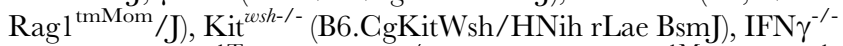
(B6.129S7-Ifng $\left.{ }^{\mathrm{tm} 1 \mathrm{Ts}} / \mathrm{J}\right), \mathrm{TCRbd}^{-/-}\left(\mathrm{B} 6.129 \mathrm{P} 2-\mathrm{Tcrb}^{\mathrm{tm} 1 \mathrm{Mom}^{-} \mathrm{Tcrd}^{\mathrm{tm1-}}}\right.$ $\mathrm{Mom} / \mathrm{J}$ ), and $\mathrm{CD} 4^{-/-}\left(\mathrm{B} 6.129 \mathrm{~S} 2-\mathrm{Cd} 4^{\text {tm1Mak}} / \mathrm{J}\right.$ ) mice (6 to 8 weeks old) were purchased from Jackson Laboratories (ME, USA) and randomly assigned into cages in groups of 6 animals. The mice were allowed free access to water and rodent diet (Harlan, Israel).

\section{Animal studies}

Yersinia strains were grown on BHIA plates (Difco) for 48 hours at $28^{\circ} \mathrm{C}$. Several colonies were suspended in a sterile saline solution, diluted to the desired infectious dose and used for the challenge. Mice (6/group, unless stated otherwise), were injected with $0.1 \mathrm{ml}$ of suspension containing the indicated amount of bacterial cells s.c. in their lower or upper back or intraperitoneally (i.p.). Bacterial cell counts were performed by serial dilution plating on BHIA. Unless otherwise indicated, the challenged animals were monitored daily for 35 days. The $F$. tularensis LVS strain was grown at $37^{\circ} \mathrm{C}$ to the mid-log phase (optical density of $0.1-0.2$ at $660 \mathrm{~nm}$ ) in TSBC (TSB [Difco] supplemented with $0.1 \%$ cysteine). The bacteria were washed and then re-suspended at the desired concentration in PBS. The bacteria were instilled i.n. $(25 \mu \mathrm{l})$ to ketamine/xylazine-anesthetized mice or injected i.p. $(0.5 \mathrm{ml})$. Experiments evaluating the ability of $Y$. pestis $\mathrm{Kim} 53 \Delta \mathrm{J}+\mathrm{P}$ to protect against $Y$. enterocolitica and $F$. tularensis were performed with OF1 outbred mice. Results from animal studies were obtained from at least two repeats.

\section{Antibody titer analysis}

Titers of anti- $F$. tularensis antibodies in serum samples were determined by ELISA in 96-well microtiter plates coated with $100 \mu \mathrm{l}$ of $10^{8} \mathrm{CFU} / \mathrm{ml}$ formalin-inactivated LVS. Antibody titers were expressed as reciprocal geometric mean titers (GMTs). The limit of detection for this assay was the reciprocal GMT value of 40 .

\section{Statistical analyses}

GraphPad statistical software was used for the statistical analyses. Survival curves were compared using the log-rank test. In all analyses, $\mathrm{P}$ values equal to 0.05 served as the limit of significance.

Table 1. Bacterial strains used in this study.

\begin{tabular}{|c|c|c|}
\hline Strains & Relevant characteristics & Reference \\
\hline \multicolumn{3}{|l|}{ Yersinia pestis } \\
\hline Kimberley53 (Kim53) & Fully virulent $Y$. pestis strain (biovar Orientalis) & {$[11,49]$} \\
\hline Kim53pGFP & Kim53 carrying pGFPuv (Clontech) & {$[50]$} \\
\hline Kim53 & yopJ-deleted Kim53 over-expressing YopP of $Y$. enterocolitica WA 0:8 & [17] \\
\hline $\operatorname{Kim} 53 \Delta \mathrm{pCD} 1 \Delta \mathrm{pPCP} 1$ & Spontaneously pPCP1 and pCD1-cured Kim53 & [17] \\
\hline EV76 & pgm- (Girard's strain) & [49] \\
\hline \multicolumn{3}{|l|}{ Yersinia enterocolitica } \\
\hline WA O:8 & Virulent $Y$. enterocolitica strain & [51] \\
\hline \multicolumn{3}{|l|}{ Francisella tularensis } \\
\hline Holarctica LVS & Live vaccine strain (ATCC 29684) & [52] \\
\hline
\end{tabular}




\section{Results and Discussion}

\section{Y. pestis over-expressing YopP activates a transient} innate protective response

In mice, the s.c. administration of $\mathrm{Kim} 53 \Delta \mathrm{J}+\mathrm{P}$ induces a highly rapid systemic resistance against $\Upsilon$. pestis strains [17]. To characterize the contribution of innate immunity to the Kim53 $\Delta J+\mathrm{P}$-activated protective response, we used immunodeficient $\mathrm{RAG}^{-/-}$mice, which lack the capacity to mount an adaptive immune response.

Parental C57BL/6J mice and their isogenic $\mathrm{RAGl}^{-/-}$mice were infected s.c. with $\mathrm{Kim} 53 \Delta \mathrm{J}+\mathrm{P}\left(10^{4} \mathrm{cfu}\right)$ or with the fully virulent $Y$. pestis strain Kim53pGFP $\left(10^{2} \mathrm{cfu}\right)$ as a control. As expected, all wild-type $\mathrm{C} 57 \mathrm{BL} / 6 \mathrm{~J}$ mice survived the $\mathrm{Kim} 53 \Delta \mathrm{J}+\mathrm{P}$ infection, whereas those infected with Kim53pGFP succumbed to the disease within seven days (Figure 1A). All immunodeficient $\mathrm{RAGl}^{-1-}$ mice infected with Kim53pGFP succumbed to the disease within nine days, whereas high survival rates of $100-89 \%$ were observed for Kim53 $\mathrm{J}+\mathrm{P}$-infected $\mathrm{RAGl}^{-/-}$mice during the initial nine days post-infection (Figure 1B). This result suggests that following the s.c. infection of $\mathrm{RAG}^{-/-}$mice with Kim53 $\Delta \mathrm{J}+\mathrm{P}$, a rapid protective innate immune response was evoked. However, the protective response was transient as the survival rate declined gradually to $14 \%$ by day 35 post-infection (mean time to death of
19 days in the Kim53 $\Delta \mathrm{J}+\mathrm{P}$-infected mice compared with 6.6 days in the $\mathrm{RAG1}^{-/-}$mice infected with Kim53pGFP).

In a subsequent cross-protection experiment, we assessed the ability of the innate immune response induced by Kim $53 \Delta \mathrm{J}+\mathrm{P}$ to provide rapid systemic protection against the challenge with a fully virulent $Y$. pestis strain inoculated s.c. at a different remote site. $\mathrm{RAG1}^{-/-}$mice were infected s.c. with $10^{4} \mathrm{cfu}$ of $\mathrm{Kim} 53 \Delta \mathrm{J}+\mathrm{P}$ and were challenged s.c. with a lethal dose of $10^{2}$ cfu of the virulent Kim53pGFP strain three days later. Indeed, the Kim53 $\Delta \mathrm{J}+\mathrm{P}-$ induced protective response in the $\mathrm{RAGl}^{-/-}$mice was also effective in postponing the time to death following Kim53pGFP infection (Figure 1C). Importantly, the survival curve of co-infected mice was statistically indistinguishable from that of mice infected with $\operatorname{Kim} 53 \Delta \mathrm{J}+\mathrm{P}$ alone $(\mathrm{P}=0.97$ by the log-rank test, Figures $1 \mathrm{~B}$ and 1C). These results indicate that a potent protective innate response can delay mouse mortality following infection with a highly virulent $T$. pestis strain and even cure a small but distinct proportion of $Y$. pestis-infected mice. One may speculate that in wild-type mice this initial delay in disease progression provides the host with the time needed to develop an effective adaptive response against $r$. pestis infection.

In an effort to characterize the distinct innate immune response induced rapidly following infection with $\operatorname{Kim} 53 \Delta \mathrm{J}+\mathrm{P}$, we compared the levels of various classes of innate immune cells in the draining inguinal lymph node (ILN) and spleen at 24 and 48 hours

A

B
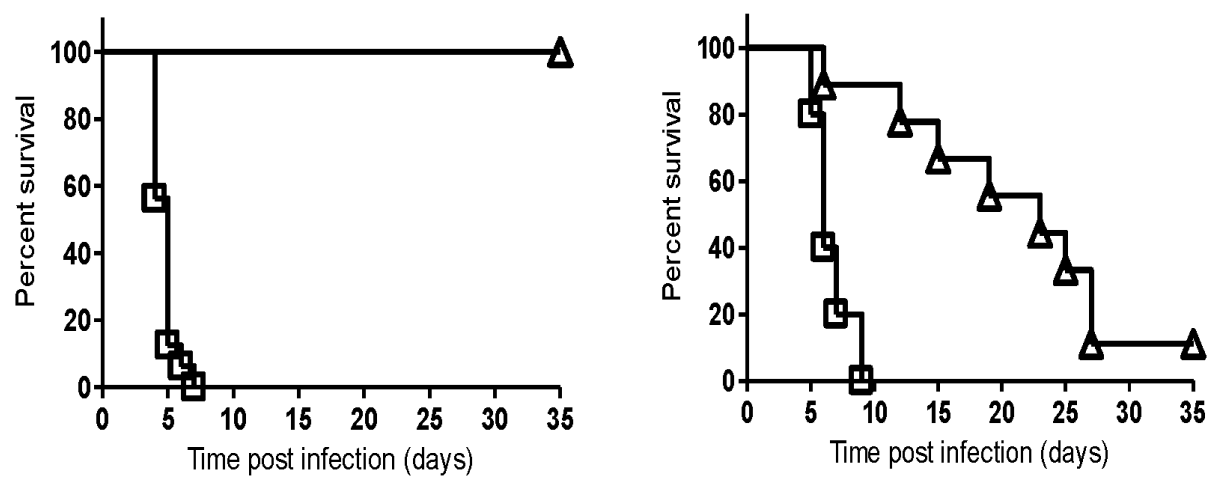

C

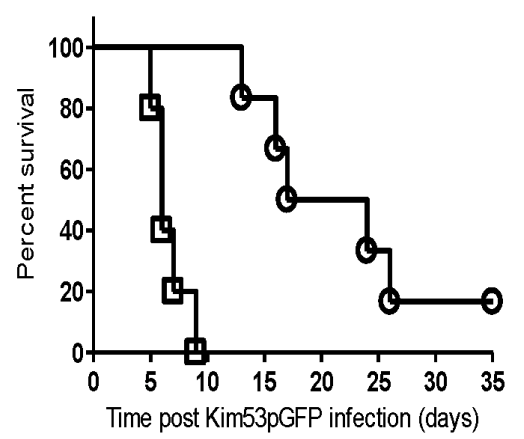

Figure 1. Transient protective immunity induced by Kim53 $3 \mathrm{~J}+\mathrm{P}$ in immunodeficient RAG $1^{-/-}$mice. $\mathrm{C} 57 \mathrm{BL} / 6 \mathrm{~J}$ mice $(\mathrm{A})$ and their isogenic $\mathrm{RAG}^{-1-}$ mice (B) were infected s.c. with either $100 \mathrm{cfu}$ of Kim53pGFP strain (square, five mice/group) or $1 \times 10^{4} \mathrm{cfu}$ of Kim53 $\mathrm{J}+\mathrm{P}$ (triangle, nine mice/ group). (C) RAG ${ }^{-1-}$ mice were either only infected s.c. with $100 \mathrm{cfu}$ of Kim53pGFP (square) or infected with $1 \times 10^{4}$ cfu of Kim53 $\mathrm{J}+\mathrm{P}$ and challenged s.c. with Kim53pGFP three days later (circle, six mice/group).

doi:10.1371/journal.pone.0083560.g001 
post-infection with $\mathrm{Kim} 53 \Delta \mathrm{J}+\mathrm{P}$ and the virulent Kim53pGFP strain using flow cytometry. However, no significant differences in the levels of neutrophils, macrophages and dendritic cells were observed between mice infected with Kim53 $\Delta \mathrm{J}+\mathrm{P}$ and Kim53pGFP (data not shown). Mast cells (MCs) are another type of pro-inflammatory cell located throughout the skin, and they are considered to be among the first cells encountered by invading pathogens. MCs can induce rapid innate immune responses through the release of mediators [23-25]. Interestingly, histamine, which is one of these MC mediators, has been shown to be important for controlling $Y$. enterocolitica infection in mice [26]. These mast cell features led us to evaluate their involvement in the specific immunity induced by s.c. infection with $\mathrm{Kim} 53 \Delta \mathrm{J}+\mathrm{P}$. However, the infection of MC-deficient mice (Kit ${ }^{\text {tesh-/-}}$ ) with $10^{4} \mathrm{cfu}$ of $\mathrm{Kim} 53 \Delta \mathrm{J}+\mathrm{P}$ via the s.c. route resulted in $100 \%$ survival. Furthermore, all Kim53 $\mathrm{J}+\mathrm{P}-$ infected $\mathrm{Kit}^{\text {wesh }-/-}$ mice were protected against the challenge with a lethal dose of the virulent Kim53pGFP strain, which was administered s.c. three days later. These results indicate that $\mathrm{MC}$ activation is dispensable for the rapid induction of protective immunity by $\mathrm{Kim} 53 \Delta \mathrm{J}+\mathrm{P}$.

\section{Interferon-gamma is essential for the rapid induction of the Kim53 $\Delta \mathrm{J}+\mathrm{P}$-mediated protective response}

Interferon-gamma (IFN $\gamma$ ) is a pleiotropic cytokine that modulates the development of both the innate and adaptive immune responses [27]. Based on the key role that IFN $\gamma$ plays in the immune system and its previously demonstrated role in the defense against Tersinia species [28-35], we evaluated the contribution of IFN $\gamma$ to $\operatorname{Kim} 53 \Delta \mathrm{J}+\mathrm{P}-$ mediated immunity. All mice infected s.c. with $10^{4} \mathrm{cfu}$ of $\mathrm{Kim} 53 \Delta \mathrm{J}+\mathrm{P}$ succumbed to the disease within four days, which was similar to mice infected with the fully virulent $Y$. pestis strain (Figure 2). Restoration of the virulent phenotype was unique to $\operatorname{Kim} 53 \Delta \mathrm{J}+\mathrm{P}$, as IFN $\gamma$-deficient mice infected with other attenuated $Y$. pestis strains, such as the vaccine strain EV76 or the $\mathrm{pCD}^{-} / \mathrm{pPCP}^{-}$derivative of Kim53, did not develop any disease symptoms (Figure 2). These observations clearly indicate that IFN $\gamma$ is a key element of $\operatorname{Kim} 53 \Delta \mathrm{J}+\mathrm{P}$-mediated immunity. Moreover, the rapid kinetics of disease progression and mortality of all $\mathrm{Kim} 53 \Delta \mathrm{J}+\mathrm{P}$-infected IFN $\gamma^{-/-}$mice within four days postinfection suggests that IFN $\gamma$ is important for the induction of protective innate responses by Kim $53 \Delta \mathrm{J}+\mathrm{P}$ prior to the development of adaptive immunity.

Numerous studies have demonstrated that $\operatorname{IFN} \gamma$ can activate innate immune cells to exert a potent antimicrobial effect against

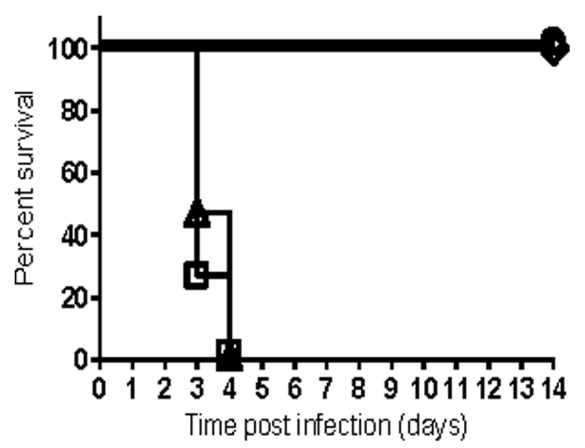

Figure 2. IFN $\gamma$ is essential for the $\mathrm{Kim} 53 \mathrm{AJ}_{+} \mathrm{P}$-mediated rapid protective response. IFN $\gamma$-deficient mice $\left(\mathrm{IFN} \gamma^{-1-}\right)$ were infected with $1 \times 10^{4}$ cfu of the following strains: Kim53 $\Delta \mathrm{J}+\mathrm{P}$ (triangle, twelve mice/ group), Kim53pGFP (square, four mice/group), EV76 (circle, four mice/ group) and Kim53 $\triangle \mathrm{pCD} 1 \triangle \mathrm{pPCP} 1$ (diamond, four mice/group). doi:10.1371/journal.pone.0083560.g002 intracellular pathogens, including Legionella pneumophila [36], F. tularensis [37,38] and Mycobacterium tuberculosis [39]. However, in the case of $\Upsilon$. pestis, the ripA gene located within the pgm locus was shown to contribute to pathogen survival in macrophages activated by IFN $\gamma$ in vitro following infection [40]. Recent in vivo studies have shown that neutralization of tumor necrosis factor alpha $(\mathrm{TNF} \alpha)$ and IFN $\gamma$ increases the lethality and bacterial burden of $\mathrm{pgm}^{-}$negative $r$. pestis strain D27 in naïve mice infected i.n. [41]. In addition, these cytokines were found to play a role in the $\mathrm{F} 1 / \mathrm{LcrV}$-mediated protective response in various mouse models of plague and are proposed to be released from $\mathrm{T}$ cells primed following vaccination $[31,34,41]$. In the present study, we further extend the observations regarding the contribution of IFN $\gamma$ to host defense against plague and demonstrate that this cytokine plays an essential role in the rapid development of protective immunity against s.c. infection with the pgm-positive Kim53 $\Delta \mathrm{J}+\mathrm{P}$ $\Upsilon$. pestis strain in naive mice (Figure 2). The rapid time course of disease progression in $\mathrm{Kim} 53 \Delta \mathrm{J}+\mathrm{P}$-infected IFN $\gamma^{-/-}$mice suggests that adaptive cellular immunity, which typically requires a longer duration to develop, is not involved in the IFN $\gamma$-mediated early protective response against $\operatorname{Kim} 53 \Delta \mathrm{J}+\mathrm{P}$. To further address this assumption, $\mathrm{TCRbd}^{-/-}$mice, which lack functional $\mathrm{T}$ cells, were infected s.c. with $10^{4} \mathrm{cfu}$ of $\operatorname{Kim} 53 \Delta \mathrm{J}+\mathrm{P}$, and survival was monitored for 35 days. All TCRbd ${ }^{-1-}$ mice survived for two weeks after s.c. infection with $10^{4} \mathrm{cfu}$ of $\mathrm{Kim} 53 \Delta \mathrm{J}+\mathrm{P}$, suggesting that $\mathrm{T}$ cells are not required for the IFN $\gamma$-mediated early protective response against $\operatorname{Kim} 53 \Delta \mathrm{J}+\mathrm{P}$ (Figure $3 \mathrm{~A})$. However, by day 35 post-infection, $50 \%$ of the $\mathrm{TCRbd}^{-/-}$-infected mice succumbed to the infection (Figure 3A), indicating that $\mathrm{T}$ cell activity is required to augment the early protective innate response against $\mathrm{Kim} 53 \Delta \mathrm{J}+\mathrm{P}$ and that this activity makes an important contribution to host defense against Kim53 $\Delta \mathrm{J}+\mathrm{P}$ infection. Notably, the ability of $\mathrm{T}$ cells to help $\mathrm{B}$ cells produce antibodies appears to play a minor role in the $\mathrm{Kim} 53 \Delta \mathrm{J}+\mathrm{P}$-mediated defense response, as $90 \%$ of $\mu$ MT B cell-deficient mice (Figure 3B) and all CD4 T celldeficient mice survived for 35 days following s.c. infection with $10^{4} \mathrm{cfu}$ of $\mathrm{Kim} 53 \Delta \mathrm{J}+\mathrm{P}$.

Currently, the exact mechanism by which IFN $\gamma$ is involved in the Kim53 $\Delta \mathrm{J}+\mathrm{P}$-mediated early innate immune response is unclear, and requires further studies. It should be noted, however, that the levels of IFN $\gamma$ measured in the spleens of mice during the first 72 hours after infection with $\operatorname{Kim} 53 \Delta \mathrm{J}+\mathrm{P}$ were similar to their levels in mice infected with the virulent Kim53pGFP strain [17], suggesting that IFN $\gamma$ might act locally following s.c. infection with $\mathrm{Kim} 53 \Delta \mathrm{J}+\mathrm{P}$. Another possibility is that the expression of IFN $\gamma$ is necessary, but not sufficient for Kim53 $\Delta \mathrm{J}+\mathrm{P}$-mediated rapid protective response, and that the ability of the host to overcome the infection depends on another, yet unknown, factor(s).

\section{Kim53 $\Delta \mathrm{J}+\mathrm{P}$ activates cross-protection against other bacterial pathogens}

Non-specific protective responses against secondary infection with heterologous bacterial pathogens have previously been described in the pioneering studies of Mackaness and Henderson in the 1960s [42,43], and in later publications by Killar [44] and Elkins [45]. Additional work by Elkin et al., also described rapid induction of non-specific, IFN- $\gamma$-mediated protection against $F$. tularensis infection using bacterial DNA [46]. The rapid activation of innate immunity by Kim $53 \Delta \mathrm{J}+\mathrm{P}$ raised the intriguing question of whether s.c. infection with Kim $53 \Delta \mathrm{J}+\mathrm{P}$ would also elicit heterologous protective immunity against other bacterial pathogens.

We first evaluated whether Kim $53 \Delta \mathrm{J}+\mathrm{P}$-mediated cross-protection could be induced against another member of the Yersinia 
A

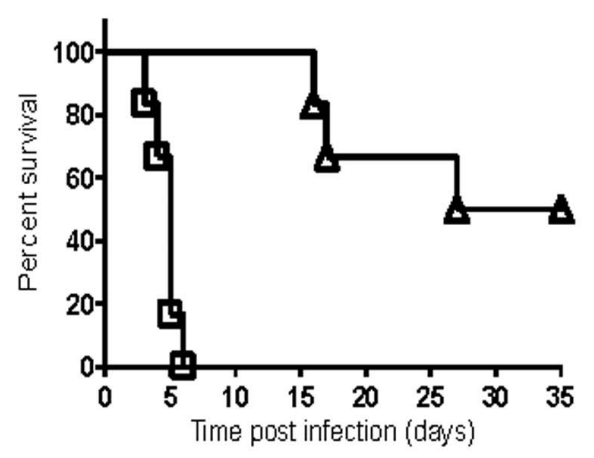

B

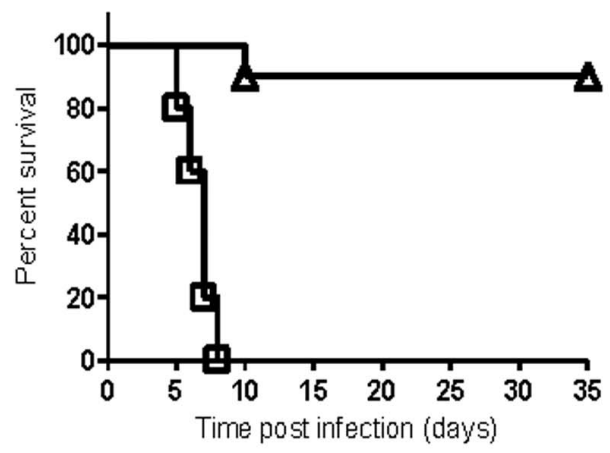

Figure 3. Contributions of $T$ cells and $B$ cells to the protective immunity induced by $\mathrm{Kim} 53 \Delta \mathrm{J}+\mathrm{P}$. $\mathrm{TCRbd}^{-/-}$mice lacking functional T cells (A) and $\mu$ MT mice lacking functional B cells (B) were infected s.c. with $10^{4} \mathrm{cfu}$ of Kim53 $\mathrm{J}+\mathrm{P}$ (triangle, ten mice/group) or $100 \mathrm{cfu}$ of Kim53 (square, five mice/group). Survival was monitored for 35 days. doi:10.1371/journal.pone.0083560.g003

genus, the food-born pathogen $Y$. enterocolitica, which causes gastroenteritis [47]. Mice were infected s.c. with $10^{5} \mathrm{cfu}$ of $\operatorname{Kim} 53 \Delta \mathrm{J}+\mathrm{P}$ and were challenged i.p. three days later with a lethal dose of $2 \times 10^{3}$ cfu of $Y$. enterocolitica WA O:8 strain. Although all mice infected with $2 \times 10^{3}$ cfu of $Y$. enterocolitica alone succumbed to the disease within 11 days, a high survival rate of $80 \%$ was observed when the mice were challenged with $Y$. enterocolitica three days after infection with $\operatorname{Kim} 53 \Delta \mathrm{J}+\mathrm{P}$ (Figure $4 \mathrm{~A}$ ). This result indicated that the Kim $53 \Delta \mathrm{J}+\mathrm{P}$-activated response can indeed provide rapid and effective cross-protection against another pathogen. However, because $r$. enterocolitica and $r$. pestis belong to the same genus and may share common antigenic determinants, we extended the evaluation of the protective immunity induced by Kim53 $\Delta \mathrm{J}+\mathrm{P}$ toward a non-related Gram-negative pathogen, $F$. tularensis, the etiologic agent of tularemia. Mice were infected i.p. and i.n. with a lethal dose of the $F$. tularensis subspecies holarctica LVS attenuated strain (500 cfu and 5,000 cfu, respectively). All infected mice exhibited disease symptoms (weight loss), and succumbed within ten days (Figures $4 \mathrm{~B}$ and $4 \mathrm{C}$ ). However, when the mice were first infected s.c. with $\operatorname{Kim} 53 \Delta \mathrm{J}+\mathrm{P}$ and challenged three days later with $F$. tularensis LVS, high survival rate of $70 \%$ was observed following the i.p. challenge and most notably, $63 \%$ survived the i.n. challenge (Figures $4 \mathrm{~B}$ and $4 \mathrm{C}$ ). In addition, substantial anti-LVS titers $\left(10^{3}-10^{5}\right)$ were measured in the sera of mice surviving the infection with $\mathrm{Kim} 53 \Delta \mathrm{J}+\mathrm{P}$ and secondary
A

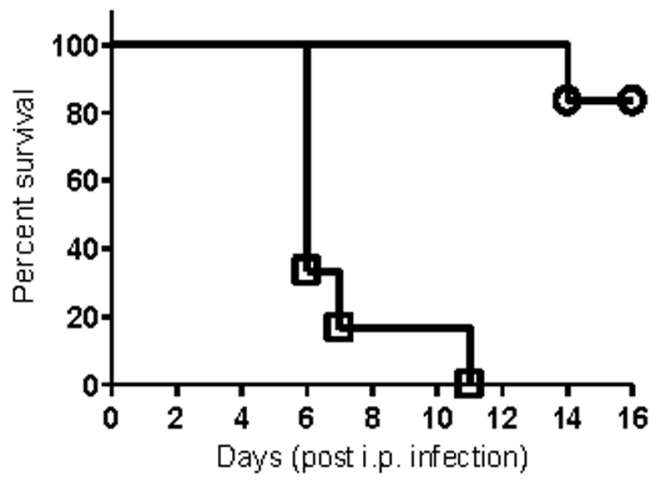

B

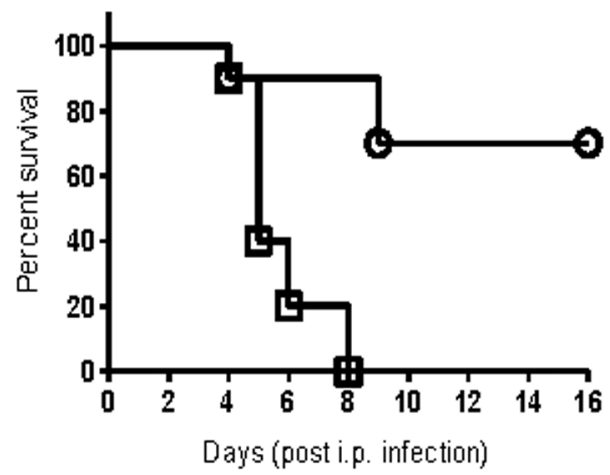

C

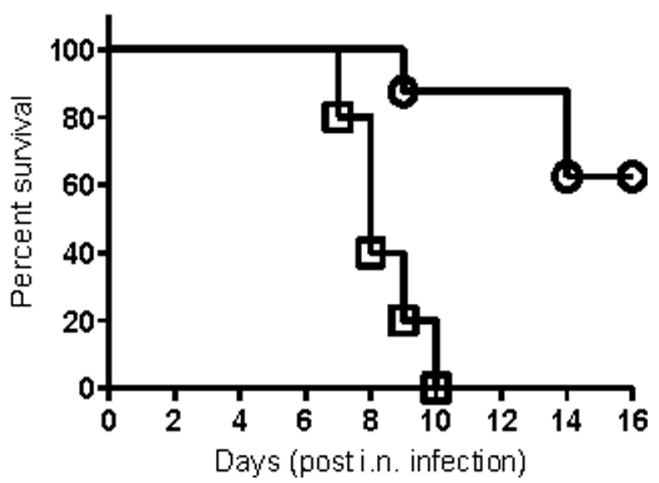

Figure 4. Kim53 $\Delta \mathrm{J}+\mathrm{P}$ induces cross-protection against $Y$. enterocolitica and $\boldsymbol{F}$. tularensis. (A) Mice were either only infected i.p. with $1 \times 10^{3}$ cfu of $Y$. enterocolitica WA (square, six mice/group) or infected s.c. with $1 \times 10^{4} \mathrm{cfu}$ of $\mathrm{Kim} 53 \Delta \mathrm{J}+\mathrm{P}$ and challenged i.p. with $1 \times 10^{3} \mathrm{cfu}$ of $Y$. enterocolitica WA three days later (circle, six mice/ group). (B) Mice were either only infected either i.p. with $500 \mathrm{cfu}$ of $F$. tularensis LVS (square, ten mice/group) or infected s.c. with $1 \times 10^{4} \mathrm{cfu}$ of $\operatorname{Kim} 53 \Delta \mathrm{J}+\mathrm{P}$ and challenged i.p. with $500 \mathrm{cfu}$ of $F$. tularensis LVS three days later (circle, ten mice/group). (C) Mice were either only infected i.n. with $5000 \mathrm{cfu}$ of $F$. tularensis LVS (square, eight mice/group) or infected s.c. with $1 \times 10^{4}$ cfu of Kim53 $\Delta \mathrm{J}+\mathrm{P}$ and challenged i.n. with $5000 \mathrm{cfu}$ of $F$. tularensis LVS three days later (circle, ten mice/group). Mortality was monitored for 16 days post-challenge. doi:10.1371/journal.pone.0083560.g004 
challenge with $F$. tularensis LVS. These findings suggest that the initial non-specific immune response evoked by $r$. pestis Kim53 $\Delta \mathrm{J}+\mathrm{P}$ delayed disease progression, allowing the development of a slower adaptive immune response against the $F$. tularensis LVS.

Notably, s.c. infection with the facultative intracellular pathogen r. pestis Kim53 $\Delta \mathrm{J}+\mathrm{P}$ induced a prompt protective response against an unrelated intracellular pathogen that can replicate in the lung, spleen and liver. One can speculate that IFN $\gamma$, which was suggested in this study to be essential for the development of rapid protection against $\mathrm{Kim} 53 \Delta \mathrm{J}+\mathrm{P}$, might also provide initial antigenindependent cross-protection against $F$. tularensis LVS due to its ability to activate macrophages. Indeed, it has been reported that rapid generation of a strong innate immune response mediated by macrophages and NK cells and dependent on IFN $\gamma$ and TNF $\alpha$, protects naïve mice against lethal infection with $F$. tularensis LVS [48].

Taken together, the present data indicate that the s.c. infection of mice with Kim $53 \Delta \mathrm{J}+\mathrm{P}$ activates an extremely rapid and potent protective response in which innate immunity appears to play a

\section{References}

1. Perry R, Fetherston J (1997) Yersinia pestis-etiologic agent of plague. Clin Microbiol Rev 10: 35-66.

2. Kool JL (2005) Risk of person-to-person transmission of pneumonic plague. Clin Infect Dis 40: 1166-1172.

3. Butler T (1983) Plague and other Yersian infections. New York: Plenum press.

4. Galimand M, Carniel E, Courvalin P (2006) Resistance of Yersinia pestis to antimicrobial agents. Antimicrob Agents Chemother 50: 3233-3236.

5. Swietnicki W, Carmany D, Retford M, Guelta M, Dorsey R, et al. (2011) Identification of small-molecule inhibitors of Yersinia pestis Type III secretion system YscN ATPase. PLoS One 6: e19716.

6. Pan N, Lee C, Goguen J (2007) High throughput screening for small-molecule inhibitors of type III secretion in Yersinia pestis. Adv Exp Med Biol 603: 367-375.

7. Garrity-Ryan LK, Kim OK, Balada-Llasat JM, Bartlett VJ, Verma AK, et al. (2010) Small molecule inhibitors of LcrF, a Yersinia pseudotuberculosis transcription factor, attenuate virulence and limit infection in a murine pneumonia model. Infect Immun 78: 4683-4690.

8. Eriksson J, Grundstrom C, Sauer-Eriksson AE, Sauer UH, Wolf-Watz H, et al. (2012) Small molecule screening for inhibitors of the YopH phosphatase of Yersinia pseudotuberculosis. Adv Exp Med Biol 954: 357-363.

9. Lin JS, Szaba FM, Kummer LW, Chromy BA, Smiley ST (2011) Tersinia pestis YopE contains a dominant CD8 $\mathrm{T}$ cell epitope that confers protection in a mouse model of pneumonic plague. J Immunol 187: 897-904.

10. Williamson ED, Oyston PC (2013) Protecting against plague: towards a nextgeneration vaccine. Clin Exp Immunol 172: 1-8.

11. Tidhar A, Flashner Y, Cohen S, Levi Y, Zauberman A, et al. (2009) The NlpD lipoprotein is a novel rersinia pestis virulence factor essential for the development of plague. PLoS One 4: e7023.

12. Smiley ST (2008) Current challenges in the development of vaccines for pneumonic plague. Expert Rev Vaccines 7: 209-221.

13. Feodorova VA, Motin VL (2012) Plague vaccines: current developments and future perspectives. Emerging Microbes \& Infections 1: e36.

14. Rosenzweig JA, Jejelowo O, Sha J, Erova TE, Brackman SM, et al. (2011) Progress on plague vaccine development. Appl Microbiol Biotechnol 91: 265286.

15. Dentovskaya SV, Kopylov PK, Ivanov SA, Ageev, Anisimov AP (2013) Molecular Bases of Vaccine Prevention of Plague. Molecular Genetics, Microbiology and Virology 28: 87-98.

16. Wang X, Zhang X, Zhou D, Yang R (2013) Live-attenuated Yersinia pestis vaccines. Expert Rev Vaccines 12: 677-686.

17. Zauberman A, Tidhar A, Levy Y, Bar-Haim E, Halperin G, et al. (2009) Yersinia pestis endowed with increased cytotoxicity is avirulent in a bubonic plague model and induces rapid protection against pneumonic plague. PLoS One 4: e5938.

18. Monack D, Mecsas J, Ghori N, Falkow S (1997) Yersinia signals macrophages to undergo apoptosis and YopJ is necessary for this cell death. Proc Natl Acad Sci U S A 94: 10385-10390.

19. Ruckdeschel K, Machold J, Roggenkamp A, Schubert S, Pierre J, et al. (1997) Yersinia enterocolitica promotes deactivation of macrophage mitogen-activated protein kinases extracellular signal-regulated kinase-1/2, p38, and c-Jun NH2terminal kinase. Correlation with its inhibitory effect on tumor necrosis factoralpha production. J Biol Chem 272: 15920-15927.

20. Zhang Y, Ting AT, Marcu KB, Bliska JB (2005) Inhibition of MAPK and NFkappaB pathways is necessary for rapid apoptosis in macrophages infected with Yersinia. J Immunol 174: 7939-7949. critical although transient role. IFN $\gamma$ was found to be a key player in mediating this early, effectively induced protective response. Consistent with the relatively low specificity characterizing the rapid induction of the innate response, $\operatorname{Kim} 53 \Delta \mathrm{J}+\mathrm{P}$-infected wildtype mice were also protected from a lethal challenge with other bacterial pathogens, including the enteropathogen $Y$. enterocolitica and the respiratory pathogen $F$. tularensis LVS. Thus, the development of new therapies to enhance the innate immune response may provide an initial critical delay in disease progression following the exposure to highly virulent bacterial pathogens such as $r$. pestis, extending the time window for successful treatment.

\section{Acknowledgments}

We wish to thank Mrs. H. Cohen for her excellent technical assistance.

\section{Author Contributions}

Conceived and designed the experiments: AZ OC EB AS EM. Performed the experiments: AZ YL YV AT EB DG MA EM. Analyzed the data: AZ EB OC GH AS EM. Wrote the paper: AZ YF GH AS EM.

21. Zauberman A, Cohen S, Mamroud E, Flashner Y, Tidhar A, et al. (2006) Interaction of Yersinia pestis with macrophages: limitations in YopJ-dependent apoptosis. Infect Immun 74: 3239-3250.

22. Brodsky IE, Medzhitov R (2008) Reduced secretion of YopJ by Yersinia limits in vivo cell death but enhances bacterial virulence. PLoS Pathog 4: e1000067.

23. Urb M, Sheppard DG (2012) The role of mast cells in the defence against pathogens. PLoS Pathog 8: e1002619.

24. Dawicki W, Marshall JS (2007) New and emerging roles for mast cells in host defence. Curr Opin Immunol 19: 31-38.

25. Abraham SN, St John AL (2010) Mast cell-orchestrated immunity to pathogens. Nat Rev Immunol 10: 440-452.

26. Handley SA, Dube PH, Miller VL (2006) Histamine signaling through the $\mathrm{H}(2)$ receptor in the Peyer's patch is important for controlling Yersinia enterocolitica infection. Proc Natl Acad Sci U S A 103: 9268-9273.

27. Schroder K, Hertzog PJ, Ravasi T, Hume DA (2004) Interferon-gamma: an overview of signals, mechanisms and functions. J Leukoc Biol 75: 163-189.

28. Autenrieth IB, Heesemann J (1992) In vivo neutralization of tumor necrosis factor-alpha and interferon- gamma abrogates resistance to Yersinia enterocolitica infection in mice. Med Microbiol Immunol 181: 333-338.

29. Nakajima R, Brubaker RR (1993) Association between virulence of Yersinia pestis and suppression of gamma interferon and tumor necrosis factor alpha. Infect Immun 61: 23-31.

30. Bohn E, Heesemann J, Ehlers S, Autenrieth IB (1994) Early gamma interferon mRNA expression is associated with resistance of mice against Yersinia enterocolitica. Infect Immun 62: 3027-3032.

31. Elvin SJ, Williamson ED (2004) Stat 4 but not Stat 6 mediated immune mechanisms are essential in protection against plague. Microb Pathog 37: 177184.

32. Pujol C, Bliska JB (2005) Turning Yersinia pathogenesis outside in: subversion of macrophage function by intracellular yersiniae. Clin Immunol 114: 216-226.

33. Parent MA, Wilhelm LB, Kummer LW, Szaba FM, Mullarky IK, et al. (2006) Gamma interferon, tumor necrosis factor alpha, and nitric oxide synthase 2, key elements of cellular immunity, perform critical protective functions during humoral defense against lethal pulmonary Yersinia pestis infection. Infect Immun 74: 3381-3386.

34. Lin JS, Park S, Adamovicz JJ, Hill J, Bliska JB, et al. (2010) TNFalpha and IFNgamma contribute to F1/LcrV-targeted immune defense in mouse models of fully virulent pneumonic plague. Vaccine 29: 357-362.

35. Zhang Y, Bliska JB (2010) YopJ-promoted cytotoxicity and systemic colonization are associated with high levels of murine interleukin-18, gamma interferon, and neutrophils in a live vaccine model of Yersinia pseudotuberculosis infection. Infect Immun 78: 2329-2341.

36. Bhardwaj N, Nash TW, Horwitz MA (1986) Interferon-gamma-activated human monocytes inhibit the intracellular multiplication of Legionella pneumophila. J Immunol 137: 2662-2669.

37. Lindgren H, Stenman L, Tarnvik A, Sjostedt A (2005) The contribution of reactive nitrogen and oxygen species to the killing of Francisella tularensis LVS by murine macrophages. Microbes Infect 7: 467-475.

38. Edwards JA, Rockx-Brouwer D, Nair V, Celli J (2010) Restricted cytosolic growth of Francisella tularensis subsp. tularensis by IFN-gamma activation of macrophages. Microbiology 156: 327-339.

39. Herbst S, Schaible UE, Schneider BE (2011) Interferon gamma activated macrophages kill mycobacteria by nitric oxide induced apoptosis. PLoS One 6: e19105. 
40. Pujol C, Grabenstein JP, Perry RD, Bliska JB (2005) Replication of Yersinia pestis in interferon gamma-activated macrophages requires ripA, a gene encoded in the pigmentation locus. Proc Natl Acad Sci U S A 102: 12909-12914.

41. Kummer LW, Szaba FM, Parent MA, Adamovicz JJ, Hill J, et al. (2008) Antibodies and cytokines independently protect against pneumonic plague. Vaccine 26: 6901-6907.

42. Mackaness GB (1964) The Immunological Basis of Acquired Cellular Resistance. J Exp Med 120: 105-120.

43. Henderson DW (1964) Mixed populations in vivo and in vitro. Symp Soc Gen Microbiol 14: 241-256.

44. Killar LM, Eisenstein TK (1985) Immunity to Salmonella typhimurium infection in $\mathrm{C} 3 \mathrm{H} / \mathrm{HeJ}$ and $\mathrm{C} 3 \mathrm{H} / \mathrm{HeNCrlBR}$ mice: studies with an aromatic-dependent live S. typhimurium strain as a vaccine. Infect Immun 47: 605-612.

45. Elkins KL, MacIntyre AT, Rhinehart-Jones TR (1998) Nonspecific early protective immunity in Francisella and Listeria infections can be dependent on lymphocytes. Infect Immun 66: 3467-3469.

46. Elkins KL, Rhinehart-Jones TR, Stibitz S, Conover JS, Klinman DM (1999) Bacterial DNA containing CpG motifs stimulates lymphocyte-dependent protection of mice against lethal infection with intracellular bacteria. J Immunol 162: 2291-2298.

47. Brubaker RR (1991) Factors promoting acute and chronic diseases caused by Yersiniae. Clin Microbiol Rev 4: 309-324.

48. Elkins KL, Rhinehart-Jones TR, Culkin SJ, Yee D, Winegar RK (1996) Minimal requirements for murine resistance to infection with Francisella tularensis LVS. Infect Immun 64: 3288-3293.

49. Ben-Gurion R, Shafferman A (1981) Essential virulence determinants of different Yersinia species are carried on a common plasmid. Plasmid 5: 183-187.

50. Ber R, Mamroud E, Aftalion M, Tidhar A, Gur D, et al. (2003) Development of an improved selective agar medium for isolation of Yersinia pestis. Appl Environ Microbiol 69: 5787-5792.

51. Mamroud E, Flashner Y, Tidhar A, Ber R, Gur D, et al. (2003) Evaluation of protective immunity induced by Yersinia enterocolitica type-III secretion system mutants. Adv Exp Med Biol 529: 425-430.

52. Bar-Haim E, Gat O, Markel G, Cohen H, Shafferman A, et al. (2008) Interrelationship between dendritic cell trafficking and Francisella tularensis dissemination following airway infection. PLoS Pathog 4: e1000211. 